Schweiz. Z. Tuberk. 1958;15:I-IV

\title{
Contents, Vol. 15, 1958
}

\section{INDEX}

Alves de Sousa, M.:

Ein Fall von bullösem Emphysem mit Sektionsbefund. (Progressive

Lungendystrophie ?) 59

Alves de Sousa, M.:

Quelques aspects anatomo-radiologiques de la tuberculose traitée par les bactériostatiques. (Tuberculomes et cavernes détergées) 221

Arnold, M.: Klinische Erfahrungen mit Viomycin-Pantothenat-Sulfat (VionactanP.) 203

Abnold, E. et Wacker, Th.:

Topographie segmentaire par tomographies obliques

Barandun, S.: vide Pult, N.

Barras, G.: Le réentraînement à l'effort chez les tuberculeux convalescents au Sanatorium Valaisan 39

Baumann, Th. : Reflexions sur la valeur comparée des tests tuberculiniques et du BCG-test 273

Bickel, G., Chauvet, M. et Miège, A.:

Erythème noueux et sarcoïdose

Birkhäuser, H.: Die Indikationen der Spital- und Sanatoriumsbehandlung sowie der häuslichen und ambulanten Therapie der Lungentuberkulose. Epide-

miologische Gesichtspunkte 246

Bochinski, K.: vide Dybicki, J.

Böhni, E.: vide Fust, B.

Borsari, G., Lauener, H. et Favez, G.:

Métabolisme de l'Isoniazide dans l'organisme en presence de PAS. Con

sequences thérapeutiques 214

Cardis, F. Preface 1

Cardis, F.: $\quad$ Les indications du traitement hospitalier (ou sanatorial), domiciliaire et ambulatoire de la tuberculose pulmonaire. Introduction 240

Cevey, M.: $\quad$ Le bactériophage dans le traitement des empyèmes pleuraux à germes aux antibiotiques 34

Chauvet, M.: vide Bickel, G.

Csànk, I. et Favez, G.:

Rapport entre l'aspect de la tuberculose pulmonaire et le titre des anticorps spécifiques circulants 196

De Chiara, C: La funzione respiratoria dopo broncografia nei malati di tubercolosi polmonare 168

Dworak, W.: vide Dybicki, J.

Dybicki, J., Bochinski, K. and Dworak, W.: 
Über einen Fall von zusätzlichem Trachealbronchus 70

Ebpecum, C. P. van and Kostyal, A.:

Chronic Substantial Emphysema in Pulmonary Tuberculosis .... 158

Fame, F. Contribution à $\Gamma$ étude de la tuberculose pulmonaire et grossesse ... 14

Favez, G.: vide Borsari, G.

Favez, G.: vide Csànk, I.:

Fourestier, M.: Sur la valeur du BCG-test 113

Fried Li, H.: Auswertung der Bronchoskopie bei der Lungentuberkulose 75

Fust, B., Böhni, E., Pellmont, B., Zbinden, G. und Studer, A.:

Experimentelle Untersuchungen mit D-Cycloserin 129

Gilbert, M.: Les indications du traitement hospitalier (ou sanatorial), domicilaire et ambulatoire de la tuberculose pulmonaire. Le point de vue du phtisio-

logue de ville 251

Gloor, H. U.: Zur Differentialdiagnose Nierendysplasie und Nierentuberkulose . . 49

GÜNTERT, H.: $\quad$ Bronchoskopische Befunde bei Atelektasen 497

Häberlin, F.: Beitrag zur Ikonographie miliarer Lungenerkrankungen 226

Hahn, J.: Behandlung der Tuberkulose mit einer molekularen Kombination von

INHundPAS , 217

Jedlicka, J.: vide Polák, E. Jedlicka, V.: vide Polák, E.

Jeker, K.: Vergleichende Untersuchung über die Verträglichkeit von oral verab-

reichtem PAS-Calcium und Benzoyl-PAS-Calcium in der Behandlung

der Lungentuberkulose 211

Jeney, E. und Zsolnai, T.:

Maleinsäure-Hydrazid und Maleinsäure-Phenylhydrazid, zwei neue

Antituberkulotika 175

Joannette, A. La clinique du BCG à Montreal 12

Kanzler, W.: Vergleichende Untersuchungen zur Tuberkulincytolyse bei Urogenitaltuberkulose 191

Kolb, R.: Statistische Untersuchungen über die Tuberkulose-Erkrankungen an

einer Schwesternschule mit besonderer Berücksichtigung der Einflüsse

auf dieselben durch die BCG-Impfung $\quad 319$

Kostyal, A.: vide Erpecum, C. P. van Lauener, H.: vide Borsari, G. Leyinský, L.: vide Polák, E.

Liebich, H.: Betrachtung einer Endemie von 74 Fallen primärer Ingestionstuber-

kulose 337

Mangen-Klein, J.: Le pronostic éloigné de quelques mauvaises indications de pneu-

mothorax 23

Miège, A.: vide Bickel, G.

Montani, S.: Faut-il maintenir la notion de tuberculose «a minima» du poumon? . 475

NlCOD, J. L.: Méningite tuberculeuse spinale sans méningite cérébrale 308

Pellmont, B.: vide Fust, B.

Picht, E.: $\quad$ Phtisiologie - médecine totale 46

Polák, E., Levinský, L., Jedlicka, J., Jedlicka, V. und 7/8Áк, F.:

Operativer Verschluß eines angeborenen Ductus æsophago-bron-

chialis bei einer Frau mit kongenitaler Lungencystose und pluriglan-

dulärer Insuffizienz: Nanosomie und Geroderma bedingt durch Ano-

varismus $\quad 92$ 
Pui/r, N. und Bahandun, S.:

Erfahrungsbericht des Team der Gesellschaft Schweizerisch $\beta \Gamma$ Tuber-

kuloseärzte zu $\gamma$ klinischen Erprobung von Cycloserin $\quad 417$

Regli, J.: $\quad$ Physiotherapie und Arbeitstraining in der modernen Heilstätten-

behandlung 234

Reibnagel, A.-M.: vide Scholer, H.

Roche, H.: Tuberculosis in Australia 2

Scholer, H. und Reibnagel, A.-M.:

Relativ-Pneumotachographie mit elektrischem Gerät 299

Steinitz, K. H.: Zur Steroidbehandlung der intrathorakalen Tuberkuloseformen, spe-

ziell der Bronchustuberkulose198

Studer, A.: vide Fust, B.

Wacker, Th. : vide Arnold, E.

Wegmann, T.: Die Lungenhistoplasmose mit besonderer Berücksichtigung der Therapie 469

Wehrlin, H.: Die Indikationen der Spital- und Sanatoriumsbehandlung sowie der häuslichen und ambulanten Therapie der Lungentuberkulose.

Der Standpunkt des Sanatoriumsarztes 258

Wipf, R.: $\quad$ La méthode des injections intracavitaires transpariétales. Quelques remarques et résultats 201

ZÁk, F.: vide Polák, E.

Zbinden, G.: vide Fust, B.

Zsolnai, T.: vide Jeney, E.

Societates Schweizerische Vereinigung gegen die Tuberkulose. Gesellschaft Schwei-

zerischer Tuberkuloseärzte. 38. wissenschaftliche Tagung in Brunnen,

26./27. April 1958. - Association Suisse contre la Tuberculose. Société

des Médecins Suisses specialises en Tuberculose. 38e reunion scienti-

fique à Brunnen, le 26/27 avril 1958187

Libri $\quad 106,182,267,414,510$

Varia IIl, 184,415

Alle Rechte, insbesondere das der Übersetzung in fremde Sprachen, vorbehalten.

Ohne ausdrückliche Genehmigung des Verlages ist es auch nicht gestattet, diesen Band Oder

Teile

daraus auf photomechanischem Wege (Photokopie, Mikrokopie) zu vervielfältigen.

(C)

Copyright 1958 by S. KaГge Г A.G., Basel

Printed in Switzerland

Cliches: Aberegg-Steiner \& Cie., AG., Bern, und Steiner \& Cie., AG., Basel

Dnick der Basler Druck- und Veriagsanstalt, Basel 